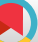

\title{
Determining the Consequences of Perceived Self-Care Ability in Stroke Patients Living at Home: A Qualitative Study in Iran
}

\author{
Nasrin Jafari-Golestan (iD ${ }^{1,2}$, Mohammadali Hosseini (iD ${ }^{3,}$, , Asghar Dalvandi (iD ${ }^{3,4}$, Masoud Fallahi- \\ Khoshknab (iD) ${ }^{5}$, Abbas Ebadi (iD) ${ }^{6,7}$, Mahdi Rahgozar (iD) ${ }^{8}$ and Souraya Sidani (iD) ${ }^{9}$ \\ ${ }^{1}$ PhD Candidate in Nursing, Department of Nursing Education, University of Social Welfare and Rehabilitation Sciences, Tehran, IR Iran \\ ${ }^{2}$ Aja University of Medical Sciences, Faculty of Nursing, Department of Nursing Management, Tehran, IR Iran \\ ${ }^{3}$ Department of Nursing Education, University of Social Welfare and Rehabilitation Sciences, Tehran, IR Iran \\ ${ }^{4}$ Department of Midwifery, Faculty of Nursing and Midwifery, Tehran Medical Sciences, Islamic Azad University, Tehran, IR Iran \\ ${ }^{5}$ Department of Nursing Education, Head of Nursing Department, University of Social Welfare and Rehabilitation Sciences, Tehran, IR Iran \\ ${ }^{6}$ Behavioral Sciences Research Center, Life Style Institute, Baqiyatallah University of Medical Sciences, Tehran, IR Iran \\ ${ }^{7}$ Nursing Faculty, Baqiyatallah University of Medical Sciences, Tehran, IR Iran \\ ${ }^{8}$ Department of Biomedical Statistics, University of Social Welfare and Rehabilitation Sciences, Tehran, IR Iran \\ ${ }^{9}$ Professor and Canada Research Chair, School of Nursing, Ryerson University, Toronto, Canada \\ "Corresponding author: Associate Professor, Department of Nursing Education, University of Social Welfare and Rehabilitation Sciences, Tehran, IR Iran. Email: \\ mahmaimy2020@gmail.com
}

Received 2019 June 23; Revised 2019 August 07; Accepted 2019 August 21.

\begin{abstract}
Background: Understanding the consequences of perceived self-care ability in stroke patients living at home is of great importance, as it is associated with continuity of care, health promotion, and improved quality of life.

Objectives: The current study aimed at determining the consequences of perceived self-care ability in stroke patients living at home in Iran.

Methods: In this qualitative study, conventional content analysis was performed. Both unstructured and semi-structured interviews were conducted with 10 patients, who had experienced chronic strokes. One interview was conducted at home, while nine others were conducted at the rehabilitation centers of Tehran, Iran. Sampling continued until reaching data saturation. All interviews were recorded, transcribed, and entered in MAXQDA. Content analysis was performed based on Graneheim and Lundman's five-step approach.

Results: Two main categories and three subcategories were extracted in this study. The two main categories included "promotion of adaptive strategies" (self-efficacy and adaptation to disability) and "maintaining a healthy lifestyle" (individual autonomy).

Conclusions: Based on the findings, the more patients are aware of their abilities and restrictions, the more they will be able to cope with the consequences of their disease during the transition from the acute to chronic phase; these patients can also adopt more suitable coping strategies.
\end{abstract}

Keywords: Perception, Self-Care, Ability, Stroke, Home, Qualitative Study

\section{Background}

Effective self-care depends on the knowledge and understanding of individuals and their self-control abilities (1). Improvement of perceived self-care ability can extend the life expectancy of patients with chronic diseases and promote the quality of life, active lifestyle, and independence (2). Generally, perceived self-care ability depends on various social determinants and health factors (1).

Stroke is recognized as an important health problem, which has spread throughout the world. It is described as one of the main causes of early morbidity and mortality, especially in low-income and middle-income countries (3).
The number of elderly people with chronic diseases is speculated to rise rapidly by 2050 (4). Self-care is an important tool in the prevention of stroke. With the increase in the elderly population around the world, the incidence of stroke is also increasing. In fact, stroke is the second leading cause of mortality worldwide (2).

The long-term lifestyle study of stroke patients suggests complex issues in their daily lives (5). In healthy people, self-care focuses on health promotion, while in people with chronic diseases, it emphasizes self-care behaviors, compliance with treatment, and healthy lifestyle (6). Today, given the increased economic burden of chronic diseases, health service providers are trying to direct care 
from hospitals to the patient's home (4).

Generally, the quality of provided care in hospitals is much higher than that provided at home (7). Therefore, attention to self-care in patients with chronic diseases and their participation in self-care activities are essential for disease management (8). Moreover, understanding the patient's self-care ability is important for determining his/her needs during discharge. In fact, by understanding the patient's perceived self-care ability, nurses and care providers can design and implement proper therapeutic interventions (9).

The patient's understanding is fundamental to his/her perceived self-care ability and independent performance. Also, therapists, by understanding the perceived self-care ability of stroke patients, can promote accurate resource allocation and planning during hospital discharge and follow-up (10). Rehabilitation centers provide nurses with the opportunity to develop interventions, which can help stroke victims cope with their chronic disease and make appropriate self-care decisions (11). Therefore, a thorough understanding of the perceived self-care ability of stroke patients living at home is very important, which can help predict the outcomes, as a significant part of the patient's performance is influenced by self-care ability.

So far, no major attempts have been made to portray the care needs of stroke patients at different stages of the disease. Also, no sufficient evidence has been presented in this area and the experiences of patients have not been discussed (12); therefore, further research is necessary. In this qualitative study, we aimed to determine the implications of understanding the perceived self-care ability of stroke patients living at home via content analysis.

\section{Objectives}

The current study aimed at determining the consequences of perceived self-care ability in stroke patients living at home in Iran.

\section{Methods}

This qualitative study was conducted using the content analysis approach in Iran in 2018. Ten independent homedwelling patients in the sub-acute and chronic phase of stroke were included in this study. One patient was interviewed at home, while nine patients were interviewed at the rehabilitation centers of Tehran, Iran. The sub-acute and chronic phase of stroke lasts more than one month.

Patients with a history of a sub-acute and chronic phase of stroke (more than one month after it) were eligible if having the ability to express oneself and willing to participate. Patients with cognitive status survey score of $<$ 21 and the functional independence measure (FIM) score of $<22$ were excluded. Patients with hemiparesis or hemiplegia, who could walk to the bathroom with a cane or walker and live at home independently, were also eligible.

In this study, the individual patient was the unit of analysis. The setting for interviewing the patients was their homes. However, most (except one) participants were reluctant to conduct interviews at home. Therefore, by referring to reputable rehab centers in the city of Tehran, the patients diagnosed with stroke were identified according to the inclusion criteria. Patients referred to the rehabilitation centers who met all inclusion criteria were selected. The researcher arranged to interview eligible patients at their convenience.

For data collection, semi-structured interviews were conducted. The researcher communicated with the participants and obtained their oral consent to participate in the study. After obtaining consent, the researcher proceeded with the interviews. The questions in the interview guide were as follows: "What is your experience of day-today living with the disease at home?" and "What do you do in terms of self-care when you are at home?" Also, the researchers asked exploratory questions during the interviews for deeper analysis (e.g., "Can you explain more?"). The duration of each interview varied from 20 to 30 minutes on average; if necessary, prolonged interviews were conducted in two stages.

Overall, 10 interviews were conducted in this study. The interviews were recorded in a quiet place and sampling continued until data saturation (13). Simultaneously, Graneheim and Lundman's five-step approach was applied to analyze the data. The interviews were immediately transcribed and entered in MAXQDA. All interviews were read to reach a thorough understanding of their content and to define the semantic units and primary codes. Next, similar primary codes were classified in larger groups and content analysis was performed (14).

In order to confirm the accuracy and reliability of data, the validity and reliability criteria proposed by Lincoln and Guba were followed. The researcher established a longterm relationship with the participants to gain their trust. After extracting the primary codes, their accuracy was confirmed by asking the participants' opinions; in the event of any conflict or inconsistency, the codes were revised. Also, the control method was implemented by two faculty members and experts in the field of qualitative research, and agreements were obtained on the selected codes and classifications.

We tried to consider the diversity of the sample in terms of age, gender, education, and marital status. Regarding ethical considerations, all participants were in- 
formed about the purpose and method of the study. After reaching an agreement, informed consent forms were obtained for participation in the study. The participants were assured of the anonymity and confidentiality of their information and audio files. They were also allowed to withdraw from the study at any time during the study.

This study was approved by the Ethics Committee of Tehran University of Social Welfare and Rehabilitation Sciences (code: IR.USWR.REC.1396.208).

\section{Results}

The participants included 10 patients with chronic stroke (two males and eight females), aged 48 - 87 years (average age, 63.7 years) (Table 1 ).

We reached data saturation by conducting 10 interviews with 10 participants and identified the primary categories. After identifying the primary themes, the primary codes were extracted from the interviews. These codes were classified after several reviews and summarized, based on their similarities. With further review and comparison, the main themes were identified as the primary categories. Two main categories and three subcategories were identified, including "promotion of adaptive strategies" (self-efficacy and adaptation to disability) and "maintaining a healthy lifestyle" (maintaining individual autonomy) (Table 2 ).

\subsection{Promotion of Adaptive Strategies}

The main extracted theme in this study was the promotion of coping strategies, which included two main subcategories of self-efficacy and adaptation to disability.

\subsubsection{Self-Efficacy}

From the point of view of the participants, the ability to perform everyday life activities, social participation, self-care, self-help, autonomy, return to work, personal hygiene, and compliance with pharmaceutical and nutritional recommendations are all the examples of perceived self-care ability. In this regard, one of the participants (male, married, 63-year-old) stated:

"After the stroke, despite hemiplegia, I was able to walk again, swim, and drive. I'm taking my medications on time, and I can bear a major share of my responsibilities on my own. Of course, I helped myself a lot to feel better."

\subsubsection{Adaptation to Disability}

From the participants' point of view, this subcategory includes accepting one's disability, use of adaptive strategies, acknowledging one's problem, feelings of helplessness and defeat related to the disease, eagerness to repeat, practice, and learn to communicate despite disability, one of the participants (male, married, 60-year-old) said:

"God has created man in a way that he can endure any difficulty. For example, it was very hard for me at first to go to the bathroom alone with only one hand... Someone had to open my belt, which was really difficult for me. I learned to do it myself over time and tried to walk on my own. Now, I can zip and unzip my pants with one hand!"

\subsection{Maintaining a Healthy Lifestyle}

This category includes one major subcategory, i.e., individual autonomy.

\subsubsection{Individual Autonomy}

From the participants' point of view, this category includes the development of self-care skills, understanding the level of dependence or independence, interest in home-based rehabilitation, and a positive outlook for maintaining independence. In this regard, one of the participants (male, married, 60-year-old) said:

"I am a dentist. After my illness, I decided to return to work, but the function of one of my hands was impaired. It was pointless to stay at home. Now, I can do simple things, like wringing prescriptions, molding, and removing dental plaques; Ifeel quite happy."

\section{Discussion}

The aim of this study was to describe the consequences of perceived self-care abilities of stroke patients living at home. The results showed that the most prominent theme is the promotion of coping strategies in stroke patients. Researchers believe that patients with high perceived selfcare ability are able to understand their disease and know how to cope with it. Individuals with high perceived selfcare ability can take the necessary measures in the event of any changes (8). Folden showed that the improvement of self-care skills can help a person cope with functional disabilities; therefore, the sustainability of stroke rehabilitation and self-care skills is important (11). Kristensen et al. believe that self-care has a very dynamic nature in patients with a chronic disease, and there is no holistic view of this concept (8). Lou et al. believe that the patient's home is the best place to continue learning and relearning the necessary skills and functional approaches for living in that environment (15).

The main theme, i.e., promotion of adaptive strategies, consists of two subcategories, self-efficacy and adaptation to disability. Self-efficacy is a psychological construct, which refers to a person's beliefs about his/her ability to produce a certain level of performance that affects his/her life (5). 


\begin{tabular}{|c|c|c|c|c|c|c|c|c|}
\hline Row & Sex & Age & Job & Education Degree & Setting & Marriage & $\begin{array}{c}\text { Duration After } \\
\text { Stroke }\end{array}$ & $\begin{array}{c}\text { Number of Stroke } \\
\text { Attacks }\end{array}$ \\
\hline $\mathbf{1}$ & Female & 73 & Housewife & Elementary & Besat & Husband died & One month & First \\
\hline 2 & Female & 87 & Housewife & Elementary & Besat & Husband died & Three years & Second \\
\hline 3 & Female & 72 & Housewife & High school & Khanevadeh & Abandoned & One year & First \\
\hline 4 & Female & 50 & Servant of the house & Diploma & Rofeideh & Living with Husband & Three months & First \\
\hline 5 & Female & 49 & Housewife & Diploma & Rofeideh & Living with Husband & Seven months & First \\
\hline 6 & Female & 48 & Housewife & Diploma & Rofeideh & Living with Husband & Three years & Third \\
\hline 7 & Female & 55 & Housewife & Illiterate & Besat & Living with Husband & 20 days & First \\
\hline 8 & Female & 76 & Emeritus teacher & Diploma & Home & Husband died & Seven months & First \\
\hline 9 & Male & 63 & Emeritus & Diploma & Rofeideh & Living with wife & Seven years & Second \\
\hline 10 & Male & 60 & Dentist & Doctor & Rofeideh & Living with wife & Seven months & First \\
\hline
\end{tabular}

Table 2. Consequences of Perceived Self-Care Ability in Stroke Patients Living at Home

\begin{tabular}{l|c}
\hline Main Category & Subcategories \\
\hline \multirow{2}{*}{ Promoting adaptive strategies } & Self-efficacy \\
\cline { 2 - 2 } & Adaptation to disability \\
\hline Maintaining a satisfying lifestyle & Maintaining individual autonomy \\
\hline
\end{tabular}

Robinson described four components of self-efficacy and their application in patients with stroke in the rehabilitation stage, including assuring patients, providing social support, having self-confidence, and showing adequate self-efficacy for rehabilitation at home (16).

Some studies have examined self-efficacy, as well as other constructs related to perceived self-care ability (17). Rabie et al. believe that if patients with chronic diseases do not have adequate knowledge of self-care, health, and autonomy-related problems, they will not be able to take care of themselves and will lose the ability to cope with their disease (18). In a combined study by Sun et al., it was found that multiple factors may influence perceived self-care ability and adaptation to disability. Some of these factors included the patient's knowledge, competence, self-esteem, education, support system, collaborative effort in home-based rehabilitation, problem-solving ability, stress management, caregiver's understanding, patient's physical condition, the patient's understanding of his/her health condition, and understanding the implications of self-care behaviors and perceived self-care ability (19). The results of these studies are consistent with the present results.

Another important theme, which is one of the implications of perceived self-care ability in patients with stroke, is maintaining a healthy lifestyle, which includes one major subcategory, i.e., individual autonomy. Based on the results of previous studies, patients who are able to maintain their individual autonomy in daily life activities and are less de- pendent on others feel more satisfied with their lives (7, 20-22). In addition, in a study by Casey et al., the level of dependence or independence was defined as a determining factor for perceived self-care ability. They claimed that understanding the experiences of stroke patients is critical for designing, revising, and implementing healthcare plans and policies to meet the needs of stroke patients (23). The findings of these studies are also consistent with the results of the present study.

\subsection{Conclusions}

A person's perceived self-care ability and autonomy are immediately evaluated by clinicians after a stroke. Stroke affects the person's ability to participate in everyday life activities, disrupts the normal course of life and consequently, reduces the quality of life. The results of previous studies show that these patients do not have a high quality of life. According to the present results, the higher the patients' understanding of their abilities and limitations is, the greater their adaptation to disability will be during the transition from the acute to chronic phase. In fact, patients with a greater understanding can adopt more suitable strategies. Therefore, the development of appropriate strategies and behaviors, such as accepting disability, selfefficacy, perseverance, spiritual practice and beliefs, cooperation of family members in the patient's adaptation, and reliance on family support can help patients better cope with their problems.

\section{Acknowledgments}

We hereby express our gratitude to all the experts and personnel of the library of the University of Social Welfare and Rehabilitation Sciences. 


\section{Footnotes}

Authors' Contribution: Study conception, searching for studies and importing them into EndNote, and participation in the analysis of studies: Nasrin Jafari-Golestan; study conception, determining how to evaluate the entered studies and final selection, and analysis of incoming studies: Mohammadali Hosseini, Asghar Dalvandi, Masoud FallahiKhoshknab, and Abbas Ebadi; revising the manuscript, and article review for plagiarism: Mohammadali Hosseini; study conception, review and final approval of the inclusion criteria, interpretation of statistical analysis methods in the included studies: Mahdi Rahgozar; Study conception, review and final approval of the inclusion criteria, final editing of the article scientifically, and checking for complying with English writing and editing rules: Souraya Sidani.

Conflict of Interests: The authors report no conflict of interest.

Ethical Approval: In line with the principles of ethical research, approval was obtained from the Research Council of the University of Social Welfare and Rehabilitation Sciences with ethical confirmation code IR.USWR.REC1396.208.

Funding/Support: No funding body contributed to the design of the study and writing of the manuscript. This doctoral thesis was not sponsored by any university or organization.

\section{References}

1. World Health Organization. Self-care in the context of primary health care Report of the Regional Consultation Bangkok, Thailand. New Delhi: World Health Organization; 2009. 2 p.

2. Kizza E, Acharya B, Maharjan R. Supporting self-care in prevention of stroke disease through patient education [dissertation]. Seinajoki University of Applied Sciences; 2014.

3. Mendis S. Prevention and care of stroke in low- and middleincome countries; the need for a public health perspective. Int $J$ Stroke.2010;5(2):86-91. doi:10.1111/j.1747-4949.2010.00406.x. [PubMed: 20446942].

4. Riegel B, Moser DK, Buck HG, Dickson VV, Dunbar SB, Lee CS, et al. Self-care for the prevention and management of cardiovascular disease and stroke: A scientific statement for healthcare professionals from the American Heart Association. J Am Heart Assoc. 2017;6(9). doi: 10.1161/JAHA.117.006997. [PubMed: 28860232]. [PubMed Central: PMC5634314].

5. Jones F, Riazi A. Self-efficacy and self-management after stroke: A systematic review. Disabil Rehabil. 2011;33(10):797-810. doi: 10.3109/09638288.2010.511415. [PubMed: 20795919].

6. Matarese M, Lommi M, De Marinis MG, Riegel B. A systematic review and integration of concept analyses of self-care and related concepts. J Nurs Scholarsh. 2018;50(3):296-305. doi: 10.1111/jnu.12385. [PubMed: 29645402].
7. Outpatient Service Trialists. Therapy-based rehabilitation services for stroke patients at home. Physiotherapy. 2003;89(3):143. doi: 10.1016/s0031-9406(05)61029-2.

8. Kristensen MAT, Holge-Hazelton B, Waldorff FB, Guassora AD. How general practitioners perceive and assess self-care in patients with multiple chronic conditions: A qualitative study. BMC Fam Pract. 2017;18(1):109. doi: 10.1186/s12875-017-0679-0. [PubMed: 29273001]. [PubMed Central: PMC5741911].

9. Thompson KO. Perceived ability for self-care: A measurement study [dissertation]. University of Maryland, Baltimore; 1992.

10. Brown T, Mapleston J, Nairn A, Molloy A. Relationship of cognitive and perceptual abilities to functional independence in adults who have had a stroke. Occup Ther Int. 2013;20(1):11-22. doi: 10.1002/oti.1334. [PubMed: 22711647].

11. Folden SL. The effect of supportive-educative nursing interventions on poststroke older adults' self-care perceptions [dissertation]. University of Miami; 1990.

12. McKevitt C, Redfern J, Mold F, Wolfe C. Qualitative studies of stroke: A systematic review. Stroke. 2004;35(6):1499-505. doi: 10.1161/01.STR.0000127532.64840.36. [PubMed: 15105517].

13. Speziale HS, Streubert HJ, Carpenter DR. Qualitative research in nursing: Advancing the humanistic imperative. Lippincott Williams \& Wilkins; 2011.

14. Graneheim UH, Lundman B. Qualitative content analysis in nursing research: Concepts, procedures and measures to achieve trustworthiness. Nurse Educ Today. 2004;24(2):105-12. doi: 10.1016/j.nedt.2003.10.001. [PubMed: 14769454].

15. Lou S, Carstensen K, Moldrup M, Shahla S, Zakharia E, Nielsen CP. Early supported discharge following mild stroke: A qualitative study of patients' and their partners' experiences of rehabilitation at home. Scand J Caring Sci. 2017;31(2):302-11. doi: 10.1111/scs.12347. [PubMed: 27481568].

16. Robinson-Smith G, Pizzi ER. Maximizing stroke recovery using patient self-care self-efficacy. Rehabil Nurs. 2003;28(2):48-51. doi: 10.1002/j.2048-7940.2003.tb02028.x. [PubMed: 12673976].

17. Godfrey CM. Self-care: A clarification of meaning and examination of supportive strategies [dissertation]. Canada: Queen's University; 2010.

18. Rabie T, Klopper HC, Watson MJ. Relation of socio-economic status to the independent application of self-care in older persons of South Africa. Health SA Gesondheid. 2016;21:155-61. doi: 10.4102/hsag.v21io.946.

19. Sun W, Doran DM, Wodchis WP, Peter E. Examining the relationship between therapeutic self-care and adverse events for home care clients in Ontario, Canada: A retrospective cohort study. BMC Health Serv Res. 2017;17(1):206. doi: 10.1186/s12913-017-2103-9. [PubMed: 28292301]. [PubMed Central: PMC5351056].

20. Stanko E, Goldie P, Nayler M. Development of a new mobility scale for people living in the community after stroke: Content validity. Aust J Physiother. 2001;47(3):201-8. [PubMed: 11552876].

21. Van Hecke A, Heinen M, Fernandez-Ortega P, Graue M, Hendriks JM, Hoy B, et al. Systematic literature review on effectiveness of selfmanagement support interventions in patients with chronic conditions and low socio-economic status. J Adv Nurs. 2017;73(4):775-93. doi: 10.1111/jan.13159. [PubMed: 27653960].

22. Mayo NE, Korner-Bitensky NA, Becker R. Recovery time of independent function post-stroke. Am J Phys Med Rehabil. 1991;70(1):5-12. doi: 10.1097/00002060-199102000-00003. [PubMed: 1994971].

23. Casey D, Murphy K, Cooney A, O'Shea E. Patient perceptions having suffered a stroke in Galway. Br J Community Nurs. 2008;13(8):384-90. doi: 10.12968/bjcn.2008.13.8.30732. [PubMed: 18856020]. 